\title{
Generalized Kinetic Theory of Electrons and Phonons: Models, Equilibrium, Stability
}

\author{
A. Rossanit and A.M. Scarfond \\ Istituto Nazionale di Fisica della Materia and \\ Dipartimento di Fisica - Politecnico di Torino \\ Corso Duca degli Abruzzi 24, 10129 Torino, Italy.
}

(Dated: February 26, 2019)

\begin{abstract}
In the present paper our aim is to introduce some models for the generalization of the kinetic theory of electrons and phonons (KTEP), as well as to study equilibrium solutions and their stability for the generalized KTEP (GKTEP) equations. We consider a couple of models, relevant to non standard quantum statistics, which give rise to inverse power law decays of the distribution function with respect to energy. In the case of electrons in a phonon background, equilibrium and stability are investigated by means of Lyapounov theory. Connections with thermodynamics are pointed out.

PACS numbers: 05.20.Dd; 05.60.Cd; 72.10.-d
\end{abstract}

Keywords: Kinetic theory; Electrons and phonons

\footnotetext{
* Corresponding author
} 


\section{INTRODUCTION}

As pointed out by Koponen, fractal or inverse power law distributions are of interest in modeling various meaningful situations in solid state physics [1]. An example, treated in ref. [1], is the thermalization of a nonequilibrium electron-phonon system.

Until recently, however, there has been little guidance on how to generalize the kinetic theory of electrons and phonons obeying non-Gibbsian statistics. In a very recent paper [2] a generalized kinetic theory for electrons and phonons has been proposed. Modified collision terms were introduced, in order to allow applications not only to electrons, but also to other particles (obeying a general statistics) which interact with a crystal lattice.

In the present paper our aim is to introduce some models for the generalization of the KTEP equations as well as to study equilibrium and stability for the GKTEP equations. We introduce a couple of models for the GKTEP, relevant to non standard quantum statistics recently proposed in the literature, and compare them from the point of view of Kinetic Theory. Both of them give rise to inverse power decays of the distribution function with respect to energy. In absence of external disturbances, it is possible to treat phonons as background at equilibrium. This assumption implies a drastic simplification of the GKTEP, since we have just to deal with the equation for electrons. Equilibrium solutions and their stability are investigated by means of Lyapunov's theory. The connection between mathematical results and Thermodynamics is shown.

First we recall the GKTEP equations and their stationary solutions. Let $N_{g}=N_{g}(\boldsymbol{k}, \boldsymbol{x}, t)$ be the distribution function of phonons (quasi-momentum $\boldsymbol{k}$, energy $\omega_{g}(\boldsymbol{k})$ of branch $g$ of the phonon spectrum and $n_{\mathbf{p}}=n(\boldsymbol{p}, \boldsymbol{x}, t)$ the distribution function of electrons [quasimomentum $\boldsymbol{p}$, energy $\epsilon(\boldsymbol{p})]$.

The generalized kinetic equations for phonons and electrons read [2]:

$$
\begin{aligned}
& \frac{\partial N_{g}}{\partial t}+\boldsymbol{u}_{g} \cdot \frac{\partial N_{g}}{\partial \boldsymbol{x}}=\left(\frac{\partial N_{g}}{\partial t}\right)_{p p}+\left(\frac{\partial N_{g}}{\partial t}\right)_{p e}, \\
& \frac{\partial n_{\mathbf{p}}}{\partial t}+\boldsymbol{v} \cdot \frac{\partial n_{\mathbf{p}}}{\partial \boldsymbol{x}}-e \boldsymbol{E} \cdot \frac{\partial n_{\mathbf{p}}}{\partial \boldsymbol{p}}=\left(\frac{\partial n_{\mathbf{p}}}{\partial t}\right)_{e p},
\end{aligned}
$$

where $\boldsymbol{u}_{g}=\partial \omega_{g} / \partial \boldsymbol{k}$ and $\boldsymbol{v}=\partial \epsilon_{\mathbf{p}} / \partial \boldsymbol{p}$. The right hand sides are given by

$$
\begin{aligned}
\left(\frac{\partial N_{g}}{\partial t}\right)_{p p}= & -\int\left\{\frac{1}{2} \sum_{g_{1} g_{2}} w_{p p}\left(\boldsymbol{k}_{1}, \boldsymbol{k}_{2} \rightarrow \boldsymbol{k}\right) \delta\left(\omega_{g}-\omega_{g_{1}}-\omega_{g_{2}}\right)\right. \\
& \times\left[\Phi\left(N_{g}\right) \Psi\left(N_{g_{1}}\right) \Psi\left(N_{g_{2}}\right)-\Phi\left(N_{g_{1}}\right) \Phi\left(N_{g_{2}}\right) \Psi\left(N_{g}\right)\right]
\end{aligned}
$$




$$
\begin{aligned}
& +\sum_{g_{1} g_{3}} w_{p p}\left(\boldsymbol{k}, \boldsymbol{k}_{1} \rightarrow \boldsymbol{k}_{3}\right) \delta\left(\omega_{g_{3}}-\omega_{g}-\omega_{g_{1}}\right) \\
& \left.\times\left[\Phi\left(N_{g_{3}}\right) \Psi\left(N_{g}\right) \Psi\left(N_{g_{1}}\right)-\Phi\left(N_{g}\right) \Phi\left(N_{g_{1}}\right) \Psi\left(N_{g_{3}}\right)\right]\right\} \frac{d \boldsymbol{k}_{1}}{8 \pi^{3}}, \\
\left(\frac{\partial N_{g}}{\partial t}\right)_{p e}= & 2 \int w_{p e}\left(\mathbf{p} \rightarrow \mathbf{p}^{\prime}, \boldsymbol{k}\right) \delta\left(\epsilon_{\mathbf{p}^{\prime}}+\omega_{g}-\epsilon_{\mathbf{p}}\right) \\
& \times\left[\varphi\left(n_{\mathbf{p}}\right) \psi\left(n_{\mathbf{p}^{\prime}}\right) \Psi\left(N_{g}\right)-\Phi\left(N_{g}\right) \varphi\left(n_{\mathbf{p}^{\prime}}\right) \psi\left(n_{\mathbf{p}}\right)\right] \frac{d \boldsymbol{p}}{8 \pi^{3}}, \\
\left(\frac{\partial n_{\mathbf{p}}}{\partial t}\right)_{e p}= & \sum_{g} \int\left\{w_{e p}\left(\boldsymbol{p}^{\prime}, \boldsymbol{k} \rightarrow \boldsymbol{p}\right) \delta\left(\epsilon_{\mathbf{p}}-\epsilon_{\mathbf{p}^{\prime}}-\omega_{g}\right)\right. \\
& \times\left[\Phi\left(N_{g}\right) \varphi\left(n_{\mathbf{p}^{\prime}}\right) \psi\left(n_{\mathbf{p}}\right)-\varphi\left(n_{\mathbf{p}}\right) \psi\left(n_{\mathbf{p}^{\prime}}\right) \Psi\left(N_{g}\right)\right] \\
& +w_{e p}\left(\boldsymbol{p}^{\prime} \rightarrow \boldsymbol{p}, \boldsymbol{k}\right) \delta\left(\epsilon_{\mathbf{p}}+\omega_{g}-\epsilon_{\mathbf{p}^{\prime}}\right) \\
& \left.\times\left[\varphi\left(n_{\mathbf{p}^{\prime}}\right) \psi\left(n_{\mathbf{p}}\right) \Psi\left(N_{g}\right)-\Phi\left(N_{g}\right) \varphi\left(n_{\mathbf{p}}\right) \psi\left(n_{\mathbf{p}^{\prime}}\right)\right]\right\} \frac{d \boldsymbol{k}}{8 \pi^{3}},
\end{aligned}
$$

where the w's are transition probabilities and electron-electron interactions have been neglected. Observe that in the limit

$$
\begin{array}{ll}
\Psi\left(N_{g}\right) \rightarrow 1+N_{g}, & \Phi\left(N_{g}\right) \rightarrow N_{g}, \\
\psi\left(n_{\mathbf{p}}\right) \rightarrow 1-n_{\mathbf{p}}, & \varphi\left(n_{\mathbf{p}}\right) \rightarrow n_{\mathbf{p}},
\end{array}
$$

the Bloch-Boltzmann-Peierls (BBP) equations [2] are recovered. The non negative functions $\Psi, \Phi$ for phonons $\left[N_{g} \in[0, \infty)\right]$, and $\psi, \varphi$ for electrons $\left(n_{\mathbf{p}} \in[0,1]\right)$ represent the arrival and departure state availability, respectively. Moreover we assume:

i) $\Phi / \Psi$ and $\varphi / \psi$ are monotonically increasing functions of $N_{g}$ and $n_{\mathbf{p}}$, respectively [2];

ii) the following conditions hold

$$
\begin{aligned}
& \varphi(0)=0, \quad \Phi(0)=0, \\
& \psi(0)=1, \quad \Psi(0)=1, \\
& \psi(1)=0, \quad \Psi(+\infty)=+\infty .
\end{aligned}
$$

Conditions (8) mean that no transition occurs when the initial state is empty, conditions (9) mean that the emptiness of the arrival state neither inhibits nor enhances a transition and conditions (10) are characteristic of fermions and bosons, respectively.

In 2] it has been shown that, implicitly, the equilibrium solutions of the generalized system 
are:

$$
\begin{aligned}
& \ln \frac{\Phi\left(N_{g}^{*}\right)}{\Psi\left(N_{g}^{*}\right)}=-\frac{\omega_{g}}{T}, \\
& \ln \frac{\varphi\left(n_{\mathbf{p}}^{*}\right)}{\psi\left(n_{\mathbf{p}}^{*}\right)}=-\frac{\epsilon_{\mathbf{p}}-\mu}{T},
\end{aligned}
$$

(* means "at equilibrium"), where $T$ is the absolute temperature of the mixture and $\mu$ is the chemical potential of the electron gas.

\section{MODELS FOR THE GENERALIZATION}

Preliminarily, consider certain particles, whose equilibrium distribution function is $f$, which obey a statistics described by the parameter $\lambda \in[-1,+1]$. Hereinafter hatted quantities are referred to these particles. In particular, for $\lambda=-1,0,+1$, we have fermions, classical particles, and bosons, respectively. Suppose, we invert this distribution function with respect to $\exp (-\mathcal{E})[$ where $\mathcal{E}=(\hat{\epsilon}-\hat{\mu}) / T]$ than we obtain:

$$
\exp (-\mathcal{E})=F(f, \lambda)
$$

In particular, for electrons $\mathcal{E}=\left(\epsilon_{\mathbf{p}}-\mu\right) / T$, for phonons $\mathcal{E}=\omega_{g} / T$, since, in this case, $\hat{\mu}=0$. The statistics of the particles we deal with is described also by the couple of function $\hat{\varphi}(f, \lambda)$ and $\hat{\psi}(f, \lambda)$, essentially positive, and obeying the following conditions:

$$
\hat{\varphi}(0, \lambda)=0, \quad \hat{\psi}(0, \lambda)=1 .
$$

In particular we have

$$
\begin{array}{ll}
\varphi\left(n_{\mathbf{p}}\right)=\hat{\varphi}\left(n_{\mathbf{p}},-1\right), & \psi\left(n_{\mathbf{p}}\right)=\hat{\psi}\left(n_{\mathbf{p}},-1\right) \\
\Phi\left(N_{g}\right)=\hat{\varphi}\left(N_{g},+1\right), & \Psi\left(N_{g}\right)=\hat{\psi}\left(N_{g},+1\right) .
\end{array}
$$

From (11) and (12) we know that at equilibrium

$$
\frac{\hat{\varphi}}{\hat{\psi}}=\exp (-\mathcal{E})
$$

Now, Eqs. (13) and (17) give

$$
\frac{\hat{\varphi}}{\hat{\psi}}=F(f, \lambda) .
$$


Let us introduce the following assumptions:

i) $\hat{\varphi} / \hat{\psi}$ is given by Eq. (18) not only at equilibrium;

ii) $\hat{\varphi}(f, \lambda)$ does not depend on $\lambda$, and we take $\hat{\varphi}(f, \lambda)=\hat{\varphi}(f, 0)$;

iii) $\hat{\psi}(f, 0)=1$.

Properties i), ii) and iii) actually hold for standard statistics (Fermi-Dirac, Maxwell-

Boltzmann, and Bose-Einstein) and, more in general, we postulate them. From Eq. (18), by accounting for $i i$ ) and $i i i)$, we get

$$
\hat{\varphi}=F(f, 0), \quad \hat{\psi}=\frac{F(f, 0)}{F(f, \lambda)} .
$$

We introduce now two models which account for an inverse power law decay of the distribution function with respect to energy:

1) The first model that we consider has been proposed by Büyükkiliç et al. [3, 4]. Relevant applications to the blackbody problem can be found in [5, 6]. Preliminarily, we define the q-deformed exponential and logarithm [7]:

$$
\exp _{q}(x)=\frac{1}{[1-(q-1) x]^{\frac{1}{q-1}}}, \quad \quad \ln _{q}(x)=\frac{1-(1 / x)^{q-1}}{q-1}
$$

The deformed quantum distribution function is given by

$$
f=\frac{1}{1 / \exp _{q}(-\mathcal{E})-\lambda}
$$

where the parameter $q$ is greater or equal to 1 (in the last case the standard statistics are recovered). Since $F(f, \lambda)=\exp \left\{\ln _{q}[1 /(1 / f+\lambda)]\right\}$, from Eq. (19) we get

$$
\hat{\varphi}=\exp \left(\ln _{q} f\right), \quad \hat{\psi}=\exp \left(\ln _{q} f-\ln _{q} \frac{f}{1+\lambda f}\right) .
$$

We have $\lim _{f \rightarrow 0} \hat{\varphi}=0$ and $\lim _{f \rightarrow 0} \hat{\psi}=1$ for $q<2$, otherwise it either vanishes $($ for $\lambda<0$ ) or it diverges (for $\lambda>0$ ),

$$
\hat{\psi}(1, \lambda)=\exp \left(-\ln _{q} \frac{1}{1+\lambda}\right)
$$

and

$$
\lim _{f \rightarrow \infty} \hat{\psi}(f, \lambda)=\exp \left(\frac{\lambda^{q-1}}{q-1}\right), \quad \text { for } \quad \lambda>0
$$



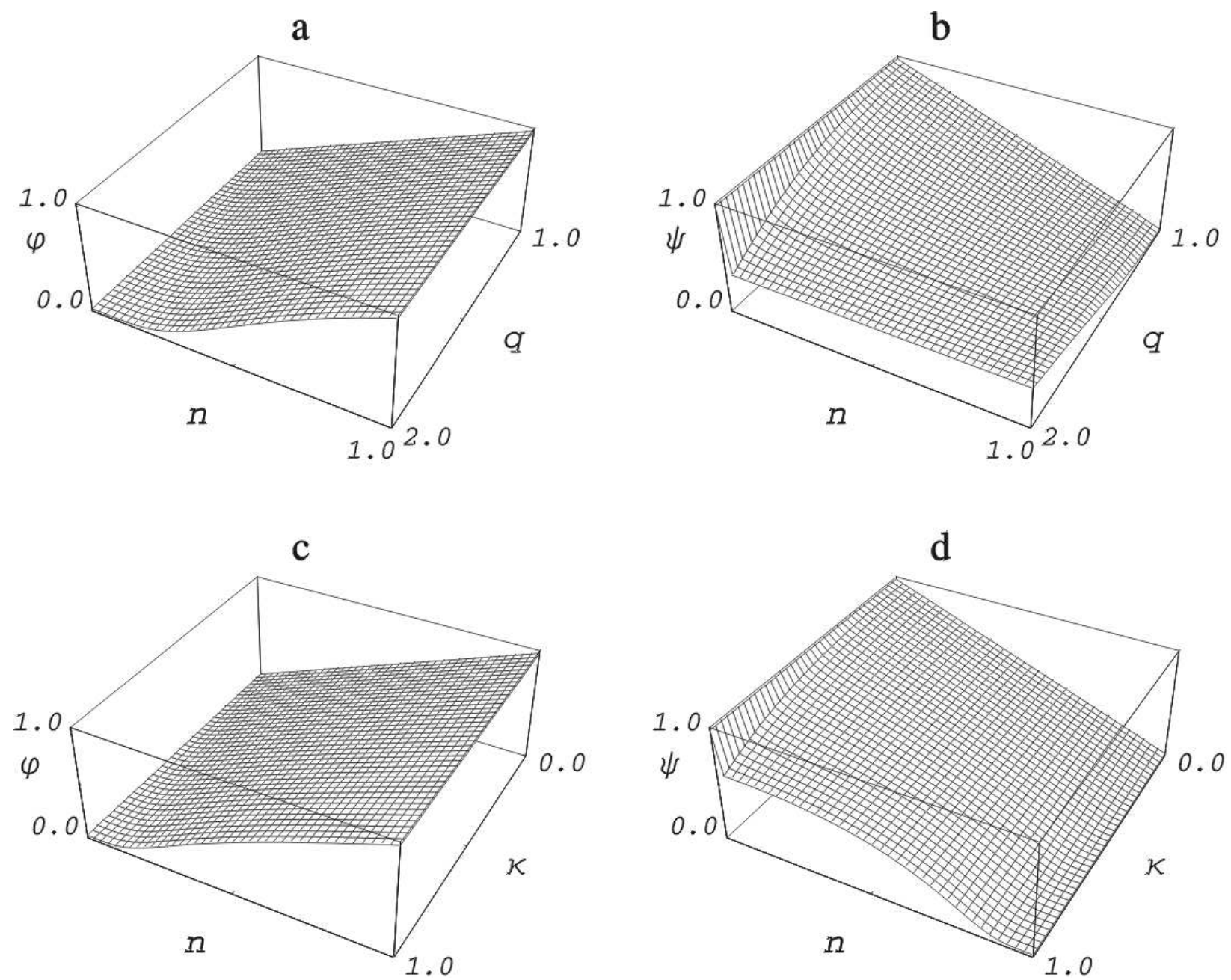

FIG. 1: a) and b): plot of $\varphi$ and $\psi$ vs $n$ and $q$ for the model of Büyükkiliç et al; c) and d): plot of $\varphi$ and $\psi$ vs $n$ and $\kappa$ for the model of Kaniadakis.

For fermions we observe that $\psi(1)=0$ is not fulfilled. Moreover, in the case of bosons the condition $\Psi(+\infty)=+\infty$ does not hold.

Figures 1a and 1b describe the couple of function $\varphi$ and $\psi$ versus both $n$ and $q$. Figures $2 \mathrm{a}$ and $2 \mathrm{~b}$ describe the couple of functions $\Phi$ and $\Psi$ versus both $N$ and $q$. Figure $1 \mathrm{~b}$ shows clearly the violation of condition $\psi(1)=0$. Both $\varphi$ and $\Phi$ start with a vanishing derivative, while both $\psi$ and $\Psi$ start with an infinite derivative.

2) Recently Kaniadakis [8, 9] has proposed a new classical distribution function, together with its quantum counterparts. An application to Bose-Einstein condensation can be found in [10]. Preliminarily, we define the new $\kappa$-deformed exponential and logarithm:

$$
\exp _{\{\kappa\}}(x)=\exp \left[\frac{1}{\kappa} \sinh ^{-1}(\kappa x)\right], \quad \ln _{\{\kappa\}}(x)=\frac{1}{\kappa} \sinh (\kappa \ln x) .
$$


Observe the remarkable property $\exp _{\{\kappa\}}(-x)=1 / \exp _{\{\kappa\}}(x)$. The deformed quantum distribution function is given by

$$
f=\frac{1}{\exp _{\{\kappa\}}(\mathcal{E})-\lambda},
$$

where the parameter $\kappa$ is taken here greater or equal to 0 (in the last case the standard statistics are recovered). Since $F(f, \lambda)=\exp \left[-\ln _{\{\kappa\}}(1 / f+\lambda)\right]$, from Eq. (19) we obtain

$$
\hat{\varphi}=\exp \left(\ln _{\{\kappa\}} f\right), \quad \hat{\psi}=\exp \left(\ln _{\{\kappa\}} f+\ln _{\{\kappa\}} \frac{1+\lambda f}{f}\right) .
$$

We have $\lim _{f \rightarrow 0} \hat{\varphi}=0$ and $\lim _{f \rightarrow 0} \hat{\psi}=1$ for $\kappa<1$, otherwise, it either vanishes (for $\lambda<0$ ) or it diverges (for $\lambda>0$ ), and

$$
\hat{\psi}(1, \lambda)=\exp \left[\ln _{\{\kappa\}}(1+\lambda)\right]
$$


FIG. 2: a) and b): plot of $\Phi$ and $\Psi$ vs $N$ and $q$ for the model of Büyükkiliç et al; c) and d): plot of $\Phi$ and $\Psi$ vs $N$ and $\kappa$ for the model of Kaniadakis. 
For electrons, the last result agrees with the condition $\psi(1)=0$. In the case of bosons, the condition for $\Phi$ when $x \rightarrow \infty$ is fulfilled as well. Figure 1c and 1d describe the couple of functions $\varphi$ and $\psi$ versus both $n$ and $\kappa$. Figures $2 \mathrm{c}$ and $2 \mathrm{~d}$ describe the couple of functions $\Phi$ and $\Psi$ versus both $N$ and $\kappa$. Figure 1d shows clearly the fulfillment of conditions $\psi(1)=0$. Both $\varphi$ and $\Phi$ start with a vanishing derivatives, while both $\psi$ and $\Psi$ start with an infinite derivative.

\section{ELECTRONS IN A PHONON BACKGROUND: EQUILIBRIUM AND STABILITY}

A usual approximation to the BBP equations consists in considering the relaxation due to p-p interactions to be much quicker than the one due to e- $\mathrm{p}$ interactions. This assumption amounts to fix, in the kinetic equation for electrons, the distribution of phonons as the equilibrium function (11) at a given temperature $T$.

The kinetic equation for electrons reads now

$$
\frac{\partial n_{\mathbf{p}}}{\partial t}+\boldsymbol{v} \cdot \frac{\partial n_{\mathbf{p}}}{\partial \boldsymbol{x}}-e \boldsymbol{E} \cdot \frac{\partial n_{\mathbf{p}}}{\partial \boldsymbol{p}}=\left(\frac{\partial n_{\mathbf{p}}}{\partial t}\right)_{e p}^{\diamond}
$$

where $\diamond$ means that $N_{g}^{*}$ has been substituted for $N_{g}$. In order to study equilibrium and its stability, it is useful to introduce the following functional:

$$
\mathcal{C}=2 \int\left(\frac{\partial n_{\mathbf{p}}}{\partial t}\right)_{e p}^{\diamond} \ln \left[\frac{\varphi\left(n_{\mathbf{p}}\right)}{\psi\left(n_{\mathbf{p}}\right)} \exp \left(\frac{\epsilon_{\mathbf{p}}}{T}\right)\right] d \boldsymbol{p}
$$

which can be rewritten as follows:

$$
\begin{aligned}
\mathcal{C}= & 2 \sum_{g} \iint w_{e p}\left(\boldsymbol{p}^{\prime}, \boldsymbol{k} \rightarrow \boldsymbol{p}\right) \delta\left(\epsilon_{\mathbf{p}}-\epsilon_{\mathbf{p}^{\prime}}-\omega_{g}\right) \ln \left(\frac{\varphi^{\prime} \psi \Phi_{g}^{*}}{\psi^{\prime} \varphi \Psi_{g}^{*}}\right) \\
& \times\left[\Phi\left(N_{g}^{*}\right) \varphi\left(n_{\mathbf{p}^{\prime}}\right) \psi\left(n_{\mathbf{p}}\right)-\varphi\left(n_{\mathbf{p}}\right) \psi\left(n_{\mathbf{p}^{\prime}}\right) \Psi\left(N_{g}^{*}\right)\right] \frac{d \boldsymbol{k} d \boldsymbol{p}}{8 \pi^{3}} \leq 0,
\end{aligned}
$$

since $(A-B) \ln (B / A) \leq 0$.

In the space homogeneous and forceless case, equilibrium solutions are given by

$$
\left(\frac{\partial n_{\mathbf{p}}}{\partial t}\right)_{e p}^{\diamond}=0
$$

\section{Proposition 1.}

Eq. (32) is equivalent to

$$
\Phi\left(N_{g}^{*}\right) \varphi\left(n_{\mathbf{p}^{\prime}}\right) \psi\left(n_{\mathbf{p}}\right)=\varphi\left(n_{\mathbf{p}}\right) \psi\left(n_{\mathbf{p}^{\prime}}\right) \Psi\left(N_{g}^{*}\right), \quad \forall \boldsymbol{p}, \boldsymbol{k}
$$


Proof. In fact, we observe that Eq. (33) $\Longrightarrow$ Eq. (32) [see Eq. (15)]. On the other hand, from Eq. (30) it follows that Eq. (32) $\Longrightarrow \mathcal{C}=0$. Tanks to Eq. (31), we obtain $\mathcal{C}=0 \Longrightarrow$ Eq. (33)

Eq. (33), by taking into account Eq. (11) and $\epsilon_{\mathbf{p}}=\epsilon_{\mathbf{p}^{\prime}}+\omega_{g}$, shows that, at equilibrium, $\ln \left[\varphi\left(n_{\mathbf{p}}\right) / \psi\left(n_{\mathbf{p}}\right)\right]+\epsilon_{\mathbf{p}} / T$ is a collisional invariant for electrons, that means $n_{\mathbf{p}}^{*}$ is given by Eq. (12). In order to study the stability of such an equilibrium solution, we introduce the following functional

$$
L=H_{e}+\frac{2}{T} \int \epsilon_{\mathbf{p}} n_{\mathbf{p}} d \boldsymbol{p}
$$

where $H_{e}=\int \mathcal{H}_{e}\left(n_{\mathbf{p}}\right) d \boldsymbol{p}$ and $\partial \mathcal{H}_{e} / \partial n_{\mathbf{p}}=2 \ln (\varphi / \psi)$ (observe that $\mathcal{H}_{e}$ is a convex function of $n_{\mathbf{p}}$, since $\varphi / \psi$ has been assumed to be monotonically increasing).

We can prove an $\mathrm{H}$ theorem for the present problem:

\section{Proposition 2.}

$L$ is a Lyapounov functional for the present problem.

Proof. By utilizing the definition of $L$ together with Eq. (29), one obtains $\dot{L}=\mathcal{C} \leq 0$, where the dot means the time derivative. Moreover, since $\left(\partial \mathcal{H}_{e} / \partial n_{\mathbf{p}}\right)^{*}=2\left(\mu-\epsilon_{\mathbf{p}}\right) / T$ by taking into account electron conservation, we have

$$
\int\left(\frac{\partial \mathcal{H}_{e}}{\partial n_{\mathbf{p}}}\right)^{*}\left(n_{\mathbf{p}}-n_{\mathbf{p}}^{*}\right) d \boldsymbol{p}=-\frac{2}{T} \int \epsilon \boldsymbol{p}\left(n_{\mathbf{p}}-n_{\mathbf{p}}^{*}\right) d \boldsymbol{p},
$$

so that we can write

$$
L-L^{*}=\int\left\{\mathcal{H}_{e}-\left[\mathcal{H}_{e}^{*}+\left(\frac{\partial \mathcal{H}_{e}}{\partial n_{\mathbf{p}}}\right)^{*}\left(n_{\mathbf{p}}-n_{\mathbf{p}}^{*}\right)\right]\right\} d \boldsymbol{p} \geq 0,
$$

due to the convexity of $\mathcal{H}_{e}$

Let us now interpret this result on a physical ground. First, we define the concentration $\mathrm{N}$, the energy density $E_{e}$, and the entropy density $S_{e}$ of the electron gas as follows:

$$
\mathrm{N}=\frac{1}{8 \pi^{3}} \int 2 n_{\mathbf{p}} d \boldsymbol{p}, \quad E_{e}=\frac{1}{8 \pi^{3}} \int 2 n_{\mathbf{p}} \epsilon_{\mathbf{p}} d \boldsymbol{p}, \quad S_{e}=-\frac{H_{e}}{8 \pi^{3}},
$$

where the factor 2 inside these integrals accounts for degeneracy. Observe that this definition of $S_{e}$ is consistent with the definitions of $T$ and $\mu$, which we have already given. In fact, at equilibrium, the following thermodynamical relationships [12] are recovered:

$$
\left(\frac{\partial S_{e}}{\partial \mathrm{N}}\right)_{E_{e}}=-\frac{\mu}{T}, \quad\left(\frac{\partial S_{e}}{\partial E_{e}}\right)_{\mathrm{N}}=\frac{1}{T} .
$$

Now, from the definition of $L$, it is easy to realize that the meaning of $\dot{L} \leq 0$ is nothing but the Clausius inequality $\dot{S}_{e} \geq \dot{E}_{e} / T$. 


\section{ACKNOWLEDGMENT}

This work has been supported by the Fonds zur Förderung der wissenschaftlichen Forschung, Vienna, under contract No. P14669-TPH.

$\dagger$ Electronic address: alberto.rossani@polito.it

‡ Electronic address: antonio.scarfone@polito.it

[1] I. Koponen, Phys. Rev. E 55, 7759 (1997).

[2] A. Rossani, Physica A 305, 323 (2002).

[3] F. Büyükkiliç, and D. Demirhan, Phys. Lett. A 181, 24 (1993).

[4] F. Büyükkiliç, D. Demirhan, and A. Güleç, Phys. Lett. A 197, 209 (1995).

[5] Q.A. Wang, and A. Le Mehaute, Phys. Lett. A 242, 301 (1998).

[6] Q.A. Wang, L. Nivanen, and A. Le Mehaute, Physica A 260, 498 (1998).

[7] S. Abe, "Nonextensive Statistical Mechanics and its Applications", Y. Okamoto editors, (Springer 2001).

[8] G. Kaniadakis, Physica A 296, 405 (2001).

[9] G. Kaniadakis, Phys. Lett. A 288, 283 (2001).

[10] G. Kaniadakis, Phys. Rev. E 66, 056125 (2002).

[11] A. Aliano, G. Kaniadakis, E. Miraldi, Physica B 325C, 35 (2003).

[12] F. Reif, "Fundamentals of Statistical and Thermal Physics", McGraw-Hill, (New York, 1965). 